\title{
OBSERVATIONS OF MULTIPLE SEISMIC EVENTS
}

\author{
By W. L. Pillant and L. Knopoff
}

\begin{abstract}
Two or more dispersed wave trains each with constant amplitude will interfere giving a resultant wave train which is amplitude modulated, if the individual waves have their principal energies in a common frequency band and if the trains arrive with time separations small compared to their total length. The dispersive characteristics of the trains need not be the same. If the component trains are of comparable magnitude, the modulation due to interference becomes significant and a "beat" phenomenon occurs. Multiple trains of dispersed seismic surface waves may occur because of a temporal and/or spatial distribution at the source or because of multipath propagation. Each of these causal mechanisms influences the amplitude and phase spectra of the resultant wave train; derived properties such as phase velocities and amplitude ratios are also influenced. In the case of multipath propagation; wavelength dependent time delays may occur. Two cases of twin earthquakes are analyzed, and the significant features of interference are demonstrated. In one case, estimates are obtained for the amplitude ratio and time delay of the second shock with respect to the first. The interpretation of seismograms and spectra influenced by multiple events is discussed.
\end{abstract}

\section{INTRODUCTION}

In surface wave phase velocity studies, a rather simple model is usually used to determine the structure of the crust and mantle. In this model an impulsive point source acts at the surface of a horizontally layered structure. A solution to this problem yields, at sufficiently large distances, a number of superimposed frequency modulated wave trains or modes whose amplitude envelopes vary relatively slowly with time. By proper filtering, each mode can be isolated for harmonic analysis. In the frequency domain, the theory predicts smoothly varying amplitude and phase spectra; these can be used in phase and group velocity determinations and in subsequent structural interpretation.

It is well known that the majority of observations of surface waves recorded by long period seismographs do not have this theoretical behavior. Excluding those events where higher modes contribute a significant proportion of the energy, it is found that many wave trains having energy only in the fundamental mode show significant amplitude modulation or "beats". It will be shown below that, in the frequency domain, these events have large minima in their amplitude spectra with corresponding discontinuities in their phase spectra. The phase discontinuities carry over into the corresponding phase velocity curves, making accurate determination of phase velocities difficult.

\section{Multiple Events-Mathematical Formulation}

Case I. Consider a seismogram consisting of the sum of two similar seismic events. Mathematically, we write the time function

$$
F(t)=f(t)+a f(t-\Delta t)
$$

where $a$ is a constant, $0 \leq a \leq 1$, and $\Delta t$ can be of either sign. As an illustration, 
consider the following example of equation (1):

$$
\begin{aligned}
F(t)= & \sin \left(t+0.5 t^{2}\right)\left[H(t)-H\left(t-T_{\max }\right)\right] \\
& +0.8 \sin \left[(t-1)+0.5(t-1)^{2}\right]\left[H(t-1)-H\left(t-1-T_{\max }\right)\right],
\end{aligned}
$$

where $H(t)$ is the unit step function, $a=0.8, \Delta t=1$, and $T_{\max }$ is a parameter chosen to terminate the disturbance after a finite time. The parameters were chosen to make the example resemble an actual seismogram with regular dispersion. The example is shown graphically in figure 1.

If we take the Fourier time transform of (1), we obtain

$$
\bar{F}(\omega)=\bar{f}(\omega)\left[1+a e^{i \omega \Delta t}\right]
$$



Fig. 1. Mathematical example of two interfering time signals.

where $\bar{F}$ is the Fourier transform of $F$ and $\bar{f}$ is the Fourier transform of $f$. For $a \ll 1$, this can be approximated by

$$
\bar{F}(\omega) \approx \bar{f}(\omega)[1+a \cos \omega \Delta t] e^{i a \sin \omega \Delta t} .
$$

When $a=1$, we have

$$
\bar{F}(\omega)=\bar{f}(\omega)[2 \cos (\omega \Delta t / 2)] e^{i(\omega \Delta t / 2)} .
$$

The spectrum of the characteristic event $f(t)$ is thus modulated in both amplitude and phase. Figure 2 shows the spectrum of the example of figure 1, and the amplitude and phase modulation can be seen. The arrows point to irregularities in the phase spectrum which correspond to minima in the amplitude spectrum.

In the example chosen here, there is a direct correlation between the minima in the amplitude spectrum and the minima of the envelope of the original time function $F(t)$. The frequencies at the beats of the time function are the frequencies of the minima in the amplitude spectrum. This need not always be the case. If the separation $\Delta t$ is large, the earliest portion of the composite time function is identical with $f(t)$. Only the later portions of the record will show envelope modulation. An extreme case would be that of two dispersive wave trains with no overlap. The amplitude spectrum of the latter composite function would have a very large num- 
ber of extrema, but there would be no interaction in the time domain. The criterion for amplitude modulation is that the simultaneous difference in instantaneous frequencies between interfering signals be small compared to their average frequency. If this is not the case, either there will be no interference or a "rider wave" effect

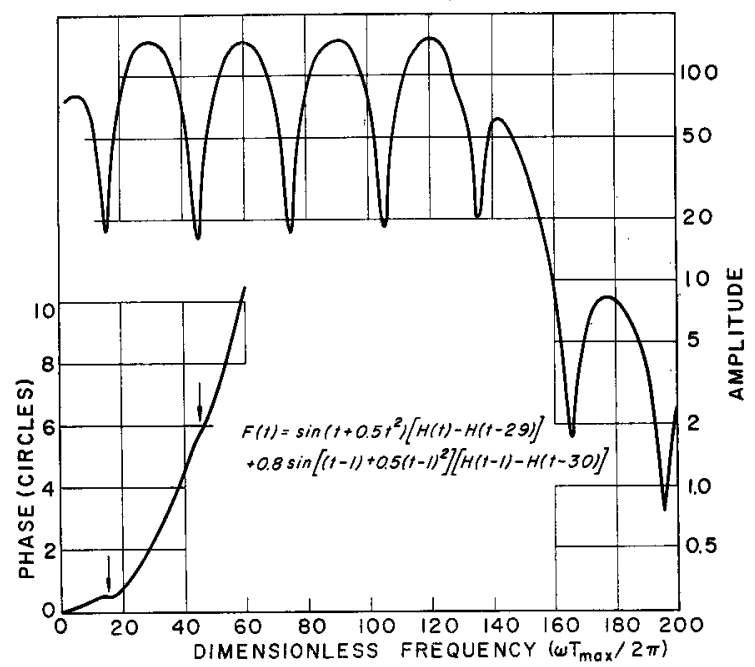

FIG. 2. Spectrum of the wave form of Fig. 1.

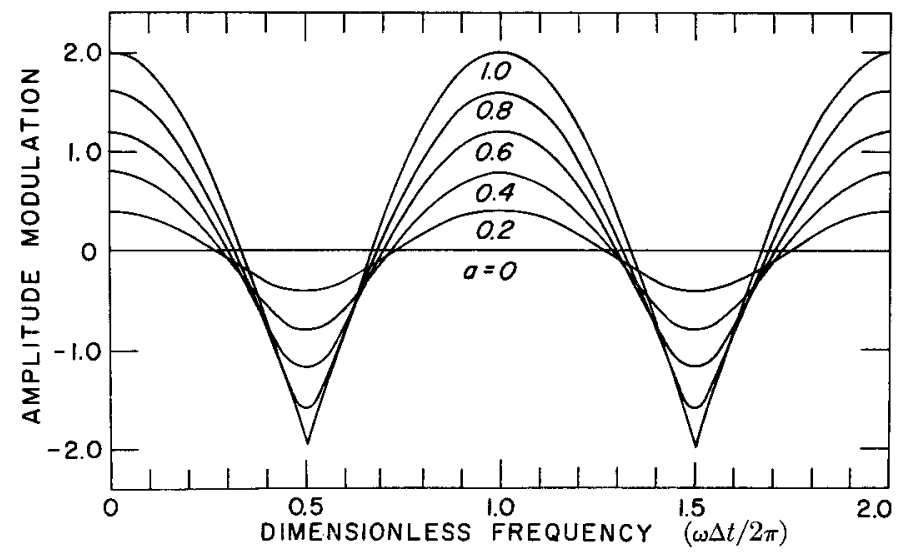

FIG. 3. Amplitude modulation of the common spectrum of two interfering signals.

The relative amplitude of the weaker signal is $a$.

will be exhibited. An example of the latter is quite often seen on long period records where crustal waves are superimposed on mantle waves. In all three cases, however, the spectrum of the composite signal will show maxima and minima as well as associated phase modulation.

Consider now the properties of the modulating term in (3),

$$
\left[1+a e^{i \omega \Delta t}\right] \text {. }
$$

Figures 3,4 , and 5 show its amplitude and phase characteristics as well as the associated time delays caused by the phase modulation. For this simple case both the 
amplitude and phase fluctuations are periodic in frequency; the magnitude of the fluctuations increases with the parameter $a$. The time delays are given by $\delta t=$ $2 \pi \delta \phi / \omega$. Here an inverse frequency dependence is shown explicitly. The largest time delays associated with the phase fluctuations occur at low frequencies or long periods. At other frequencies, large phase fluctuations and the corresponding time



Fig. 4. Phase modulation of the common spectrum of two interfering signals. The relative amplitude of the weaker signal is $a$.



FIg. 5. Relative time delay due to phase modulation of a common spectrum of two interfering signals. The relative amplitude of the weaker signal is $a$.

delays occur in the regions of minimum amplitude of the amplitude modulating factor. The parameter $a$ and the maximum phase error $\phi_{\max }$ (in circles) are related through the expression

$$
a=\sin 2 \pi \phi_{\max }
$$


Consider also the autocorrelation of the function of equation (1).

$$
\begin{aligned}
R(\tau) & =\int_{-\infty}^{\infty} F(t) F(t+\tau) d t / \int_{-\infty}^{\infty} F^{2}(t) d t \\
& =\frac{\left(1+a^{2}\right) R_{0}(\tau)+a\left[R_{0}(\tau-\Delta t)+R_{0}(\tau+\Delta t)\right]}{\left(1+a^{2}\right)+2 a R_{0}(\Delta t)}
\end{aligned}
$$



FIG. 6. Autocorrelation of two interfering signals with different frequency bandwidths and of a simple frequency modulated signal.

where

$$
R_{0}(\tau)=\int_{-\infty}^{\infty} f(t) f(t+\tau) d t / \int_{-\infty}^{\infty} f^{2}(t) d t
$$

and $\tau$ is the lag time. This evaluation is performed using appropriate limiting procedures if the integrals do not exist. Equation (7) shows that if $R_{0}(\tau)$ is highly peaked for small $\tau$, then $R(\tau)$ will have additional peaks at the positions $\tau= \pm \Delta t$. On the other hand, if $R_{0}(\tau)$ is blunted and has relatively large side lobes, then the additional peaks of $R(\tau)$ will be difficult to distinguish.

In the example chosen,

$$
f(t)=\sin \left(t+0.5 t^{2}\right)\left[H(t)-H\left(t-T_{\max }\right)\right],
$$

the instantaneous frequency varies linearly with time. Thus the spectral bandwidth is a linear function of $T_{\text {max }}$. Figure 6 shows three autocorrelation functions 
demonstrating the properties of (7). The upper curve is the autocorrelation function of $F(t)$ in (2), with $T_{\max }=14$; for the middle curve $T_{\max }=29$. It can be seen that, with a bandwidth of approximately twice that for the first curve, the peak of the second curve at $\tau=1$ is much sharper. The last curve is the autocorrelation function of $f(t)$ in (8) with $T_{\max }=29$. The additional peak stands out clearly in the first two cases.

Case II. Consider the case where the two events are not quite the same, i.e.,

$$
F(t)=f(t)+g(t-\Delta t)
$$

where $g(t)$ differs slightly from $f(t)$, and again $f(t)$ is the more energetic event. Then we write

$$
\vec{F}(\omega)=\bar{f}(\omega)\left[1+\{\bar{g}(\omega) / \bar{f}(\omega)\} e^{i \omega \Delta t}\right]
$$

If $\bar{g}(\omega) / \bar{f}(\omega)$ is a slowly varying function of frequency, both in amplitude and phase, compared with $e^{i \omega \Delta t}$, again amplitude and phase modulation occur, but this time they are not periodic unless $\bar{g}(\omega)$ and $\bar{f}(\omega)$ have the same spectral characteristics.

Case III. Consider the situation when the interfering signal is composed of not one or more similar time functions, but is a continuous superposition of similar events, i.e.,

$$
F(t)=\int_{-\infty}^{\infty} S\left(t^{\prime}\right) f\left(t-t^{\prime}\right) d t^{\prime}
$$

where $S\left(t^{\prime}\right)$ represents the amplitude distribution in time of similar events and $f(t)$ represents the characteristic event. In this case we have

$$
\bar{F}(\omega)=\bar{S}(\omega) \cdot \bar{f}(\omega)
$$

Again the characteristic spectrum $\bar{f}(\omega)$ is modulated by the factor $\bar{S}(\omega)$. If, for example, a uniform continuum of events were observed over an interval of length $T$, i.e.,

$$
S(t)=[H(t+T / 2)-H(t-T / 2)]
$$

then the modulation function $\bar{S}(\omega)$ has the form

$$
\bar{S}(\omega)=\frac{\sin \omega T}{\omega T}
$$

Again amplitude minima and appropriate phase fluctuations occur at the zeros of $\bar{S}(\omega)$. This type of modulation may or may not be periodic depending upon the spectral properties of $S(t)$. Ben-Menahem (1961) and Ben-Menahem and Toksöz 
$(1962,1963 \mathrm{a}, 1963 \mathrm{~b})$ have made extensive investigations of modulation of a similar type; their composite signal was generated by a moving source.

Case IV. Consider the function

$$
F(t)=\int_{-\infty}^{\infty} A(k)\left[e^{i k x_{1}}+a e^{i k x_{2}}\right] e^{-i \omega t} d \omega
$$

This represents a pulse which has traveled over two separate paths with different lengths but with the same transmission characteristics. Taking the time transform of (13) we find that

$$
\bar{F}(\omega)=A(k) e^{i k x_{1}}\left[1+a e^{i k\left(x_{2}-x_{1}\right)}\right] .
$$

If the propagation is in a dispersive medium where $k=\omega / c(\omega)$ and $c$ is the phase velocity, then

$$
\bar{F}(\omega)=A(k) e^{i k x_{1}}\left[1+a e^{i \omega\left(x_{2}-x_{1}\right) / c(\omega)}\right]
$$

Again there is a modulation in the frequency domain, but it is non-periodic because of the factor $c(\omega)$.

If the propagation characteristics of the paths are dissimilar and/or if frequency dependent reflection or refraction coefficients are present, the ratio $a$ may become complex with amplitude and phase which are functions of the frequency. Even more complicated spectra may arise when more than two events are considered as in Case III.

\section{Multiple Events-Observations}

It is well known that only a small proportion of observed teleseisms have a smoothly modulated envelope. We can demonstrate that in two cases the characteristics of multiple events are clearly discernible and contribute almost all the "beat" to the time record. This does not imply, however, that all "beats" are due to multiple events. On the other hand, it is difficult to think of any reasonable mechanism which would give absorption bands over the entire spectrum with varying spacings or with occasionally periodic spacings.

The first case is the earthquake pair which occurred in California: 9 April 1961,

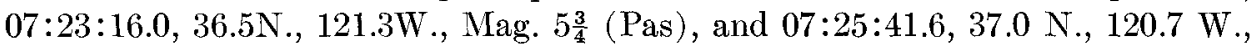
Mag. $5 \frac{1}{2}$ (Pas), Hollister, California; U.S.C.G.S. coordinates. The digitizations of the time series recorded at four stations (Palisades, N. Y.; Chur, Switzerland; Oropa, Italy; and Stuttgart, Germany) are shown in Figure 7. The European stations had identical instrumentation of $15-90$ vertical Press-Ewing seismographs; the record at Palisades was recorded by their 30-100 vertical instrument. Amplitude spectra for the four stations are shown in figure 8 . The time difference for the two events is listed as 146 seconds. In figure 8, the minima in the spectra corresponding to a time difference of 146 seconds are marked by arrows. Figure 9 shows the autocorrelation functions of the time series at the four stations with a lag of 


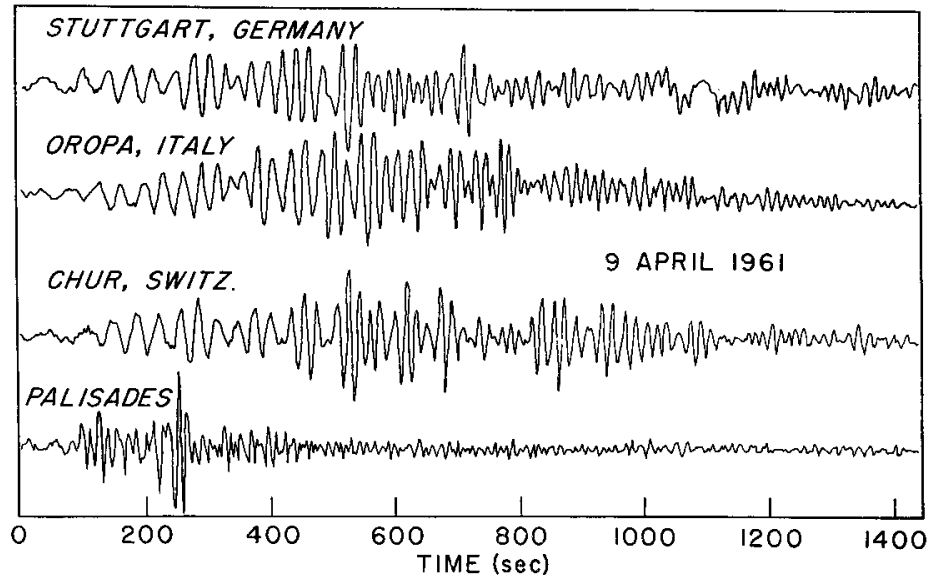

FIg. 7. Digitized time series for the event of 9 April 1961.

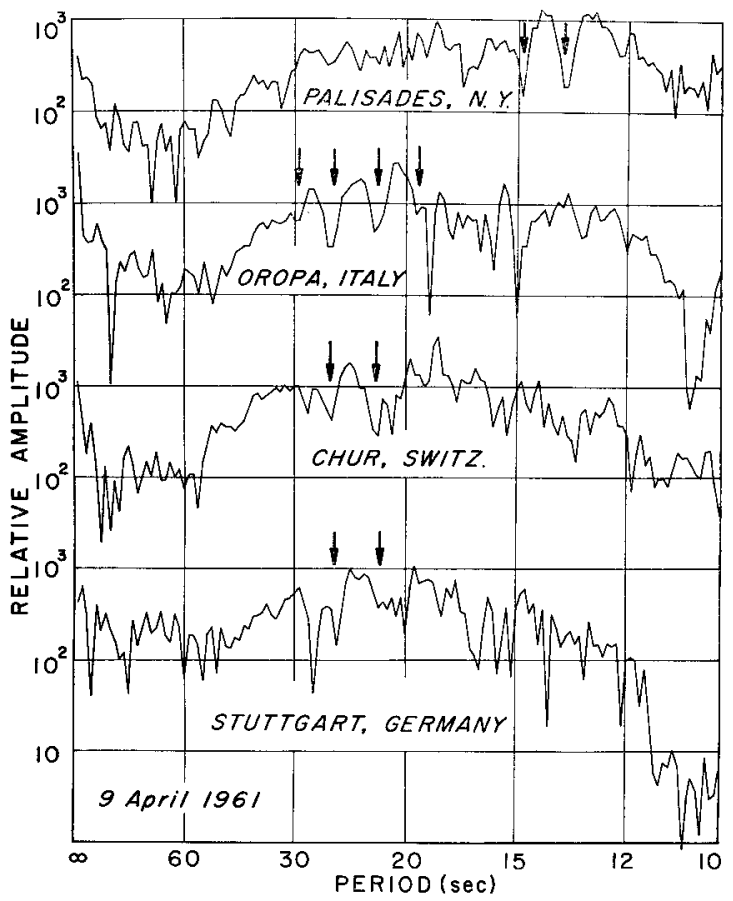

Fig. 8. Spectral amplitudes for the event of 9 April 1961.

146 seconds indicated by an arrow. The side peaks are diffused and do not give a good estimate of the time separation of the two events.

The second case is the earthquake pair which occurred in the Aleutian Islands: 14 June 1962, 07:51:51.0, 54.3 N., 169.1 E., Mag. 6 6 $\frac{1}{4}$ (Pas), and 07:55:48.9, 54.2 N., 169.3 E., Mag. 6 (Pas); Near Islands, U.S.C.G.S. coordinates. Tracings of the seismograms recorded at three stations (Mineral, California; Mt. Shasta, 
California; and Klamath Falls, Oregon) are shown in figure 10. Identical 15-90 vertical seismographs were located at the three stations. Amplitude spectra are shown in figure 11; the modulation of the spectra is clearly visible. The ratios of the maxima and minima of the spectral amplitudes range from two to three, corresponding to an amplitude ratio $a$ between the second and first events of 1:3 to $1: 2$.



FIG. 9. Autocorrelations for the event of 9 April 1961.

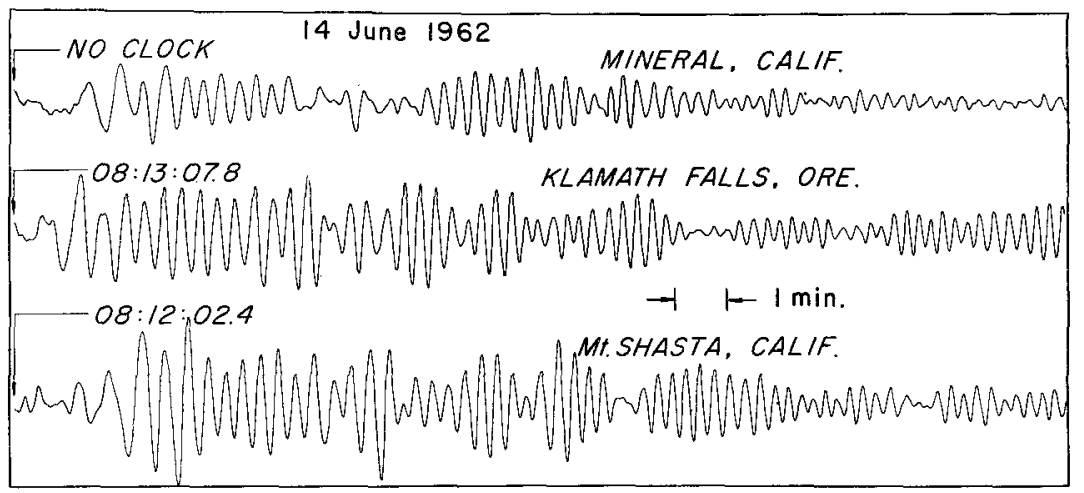

FIG. 10. Seismogram tracings for the event of 14 June 1962.

Spacings corresponding to a time difference of 238 seconds are indicated by arrows. Figure 12 shows the autocorrelations, again with a time lag of 238 seconds indicated. If the time lag were computed from the data of figures 11 and 12 a minimum uncertainty of $\pm 5 \%$ or 12 seconds would be obtained. The use of this method to obtain the time interval between shocks leads to results which are not as precise as those of direct observation on body waves.

Because of the relatively large time separation of these two events, a number of additional operations could be carried out. In the first of these, numerical filtering by several band pass filters was performed. In the band $40-200$ seconds, two Ray- 
leigh pulses were clearly visible at each station. The ratio of the amplitude of the second to the first was: Mineral, 0.38; Klamath Falls, 0.33; and Mt. Shasta, 0.40.

In a second operation, filtering by a numerical filter whose center frequency and bandwidth varied in time was carried out. This eliminated a large part of the



FIG. 11. Spectral amplitudes for the event of 14 June 1962.

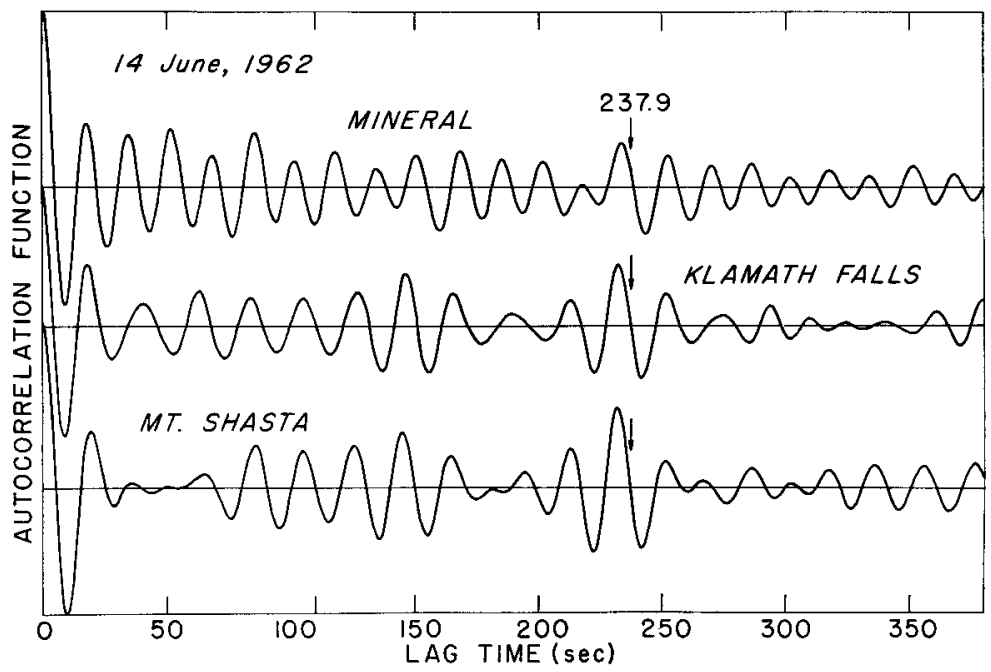

FIG. 12. Autocorrelations for the event of 14 June 1962. 
second event whose corresponding frequencies should have appeared 238 seconds later. The time-frequency relationships of the events and of the filter are indicated by figure 13. Again a spectral analysis was performed, and the spectra of the three stations are shown in figure 14 . The modulation has been removed at the lower frequencies. Phase differences between the spectra of the raw time series and the spectra of the smoothed time series have been plotted in figure 15. The peak-topeak amplitude of the phase differences lies between 0.06 and 0.10 . This corresponds to an amplitude ratio between second and first events of 0.37 to 0.59 . The

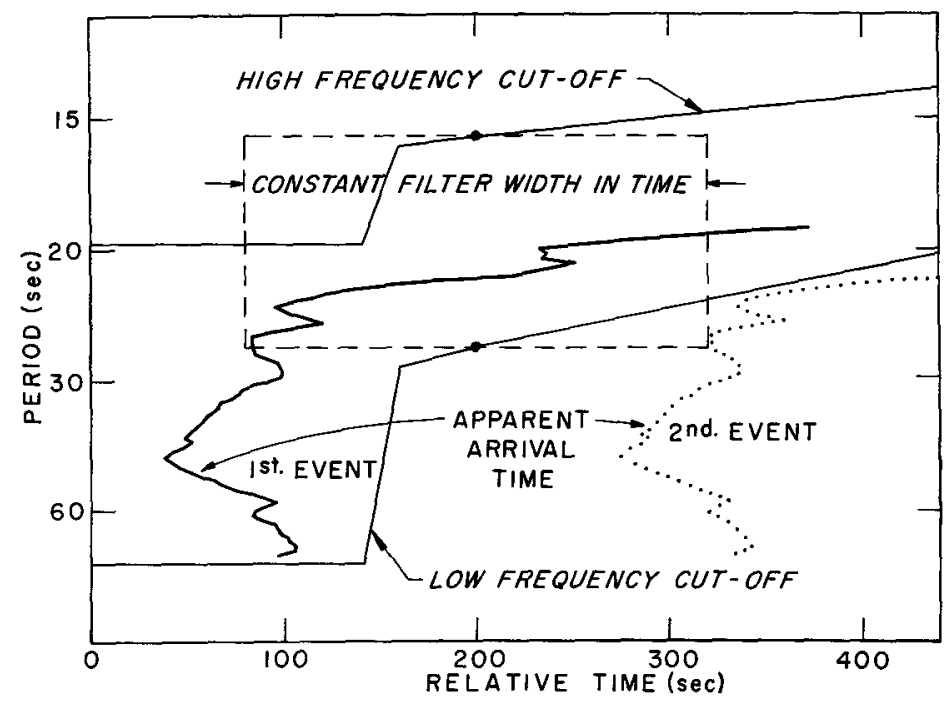

FIg. 13. Frequency-time relationships for the two events of 14 June 1962. A variable filter with characteristics given by the boxed area was constructed to reject the second event.

ratio of the amplitudes of the two events can be summarized as follows:

\begin{tabular}{|c|c|}
\hline Method & Interval of amplitude ratio \\
\hline Pasadena magnitudes (U.S.C.G.S.) & 0.56 to 1.0 \\
\hline Filtered Rayleigh wave pulses....... & 0.33 to 0.40 \\
\hline Amp. spectra extremum ratio........ & 0.33 to 0.50 \\
\hline Phase spectra extremum amplitude... & 0.37 to 0.59 \\
\hline
\end{tabular}

There is fair agreement between the last three ratios where the separation of events was performed mathematically, whereas the first is somewhat higher. This may be due to difficulty in adequately separating the two events visually. Even so, the uncertainties in the last three values are sufficiently large that the results are primarily qualitative. In the case of the Hollister, California events, similar numbers could not be extracted from the data.

Although the variable filter technique has removed almost all of the extrema of the amplitude spectra due to source effects, there remains a large minimum at 32 




FIG. 14. Spectral amplitudes for the filtered time series for the event of 14 June 1962.



Fig. 15. Phase modulation due to two shocks for the event of 14 June 1962.

seconds for Mineral and minima at 22 seconds at Klamath Falls and Mt. Shasta indicated by arrows in figure 14. In figure 11 it is seen that these minima are superimposed upon those due to the dual shock. Inasmuch as these minima are not common to all the stations, it is likely that they reflect some property of the path 
to the individual station; a study of the influence of path upon the interference is presented below.

Lastly, according to the simplified theory introduced above, surface waves emanating from a complex source should have nearly the same characteristics at several stations all far from the source and near to exch other, i.e., only a few wavelengths apart. For widely separated stations there may be azimuthal effects which will make the character of the records quite different from station to station (see, for example, Ben-Menahem, 1961). In the case of the pair of events in the Near Islands, phase differences were taken between the three pairs of stations using the unfiltered data. Figure 16 exhibits these phase differences. It is evident that most

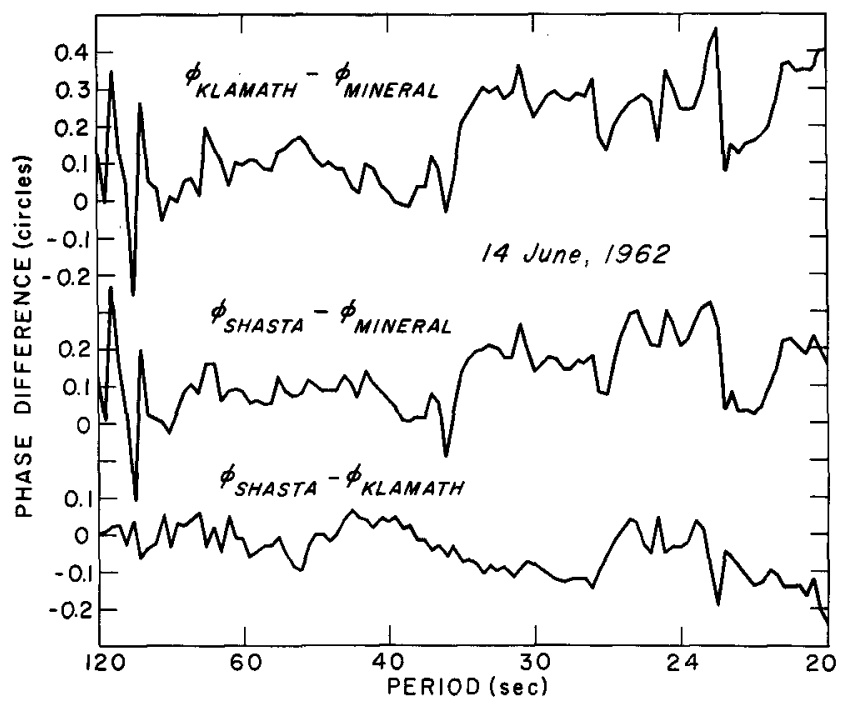

Fig. 16. Phase differences between stations for the event of 14 June 1962.

of the modulation due to the pair of shocks is eliminated, but the resulting differences are by no means smooth. Particularly evident is a discontinuity at 32 seconds due to the recording at Mineral. The large scatter is due to contamination from digitization errors and other unknown sources.

In summary, we can say that in the case of two earthquakes of similar magnitude occurring at about the same time, interference takes place as indicated by the theory. One mechanism causing records with "beats" has been demonstrated. However, as indicated by the examples above, the converse problem is difficult. Even if a regular pattern were observed at all stations, indicating a common effect due to a complex source, it is difficult to assign accurate values to the parameters involved. Hence the use of this type of interference phenomenon as a diagnostic aid is limited.

In the preceding example, it was shown that the effects associated with a complex focal mechanism can be at least partially eliminated. Yet, in figure 14, the amplitude spectrum of Mineral has a large minimum which is not common to the other two stations. Since all three stations are on approximately the same azimuth, this effect 
is probably not due to a frequency dependent source directivity. Therefore, it is reasonable to assume that this effect is due to interference caused by signals arriving over a discrete or a continuous set of multiple paths.

If the selective interference is due to multipath propagation, it would be ex-



FIG. 17. Digitized time series for the event of 9 March 1961.



FIG. 18. Spectral amplitudes for the event of 9 March 1961. 
pected to occur most frequently in areas of complex structure. This was borne out by observations made with a network of four 15-90 Press-Ewing vertical seismographs stationed in and about the Alps during the period September 1960 to April 1961. The spectra of almost every seismic event analyzed had large minima which were not common to all stations. Only teleseisms coming from the northwest seemed to be relatively free from interference at all stations. This direction of approach is generally perpendicular to the continental margin and to the Alpine structure.

Figure 17 shows a typical set of digitizations of an event observed at the four stations (Besançon, France; Chur, Switzerland; Oropa, Italy; and Stuttgart, Germany) and whose epicenter was on the mid-Atlantic ridge. The event is given as 9 March 1961, 03:59:08.7, 10.9 N., 41.7 W., U.S.C.G.S. coordinates. Only data

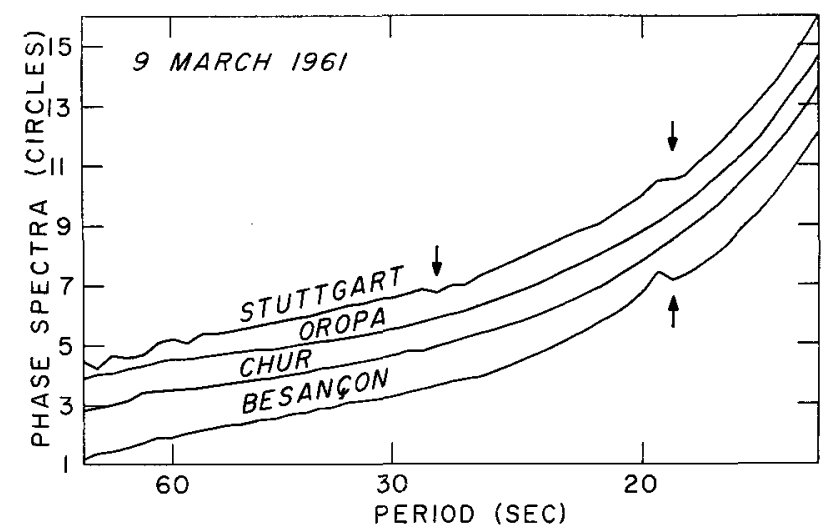

Fig. 19. Spectral phases for the event of 9 March 1961.

for Besançon and Stuttgart show rather severe interference of some type, while those for Chur show a small interference pattern and no interference is visible on the Oropa record. These recordings are not uniformly characteristic of the site. In other examples, Oropa was seen to exhibit the most interference and Stuttgart the least, and so on. On the other hand, there is a strong repeatable azimuth effect at any one station.

Amplitude spectra for the four records are given in figure 18. The spectra show significant noise at the wings of the curves; in the center, the signal spectrum is well above the noise in a band from 15 to 60 seconds. In this period range, the amplitude spectrum for Oropa is the smoothest, and the smoothness of the curves deteriorates to the rather poor quality in those for Besançon and Stuttgart. In fact, the quality of the time records and the relative absence of beats is highly correlated to the degree of smoothness of the spectrum. Figure 19 shows the corresponding phase spectra with discontinuities at 19 and 28 seconds indicated by arrows. It might be pointed out here that, in the authors' experience, phase fluctuations always imply amplitude fluctuations, but the converse is not always true: the amplitude minimum at 28 seconds at Besançon has little or no corresponding phase fluctuation.

Other workers have presented data which show similar effects. Pomeroy (1963) and Toksöz, Ben-Menahem, and Harkrider (1963) have investigated spectra from 


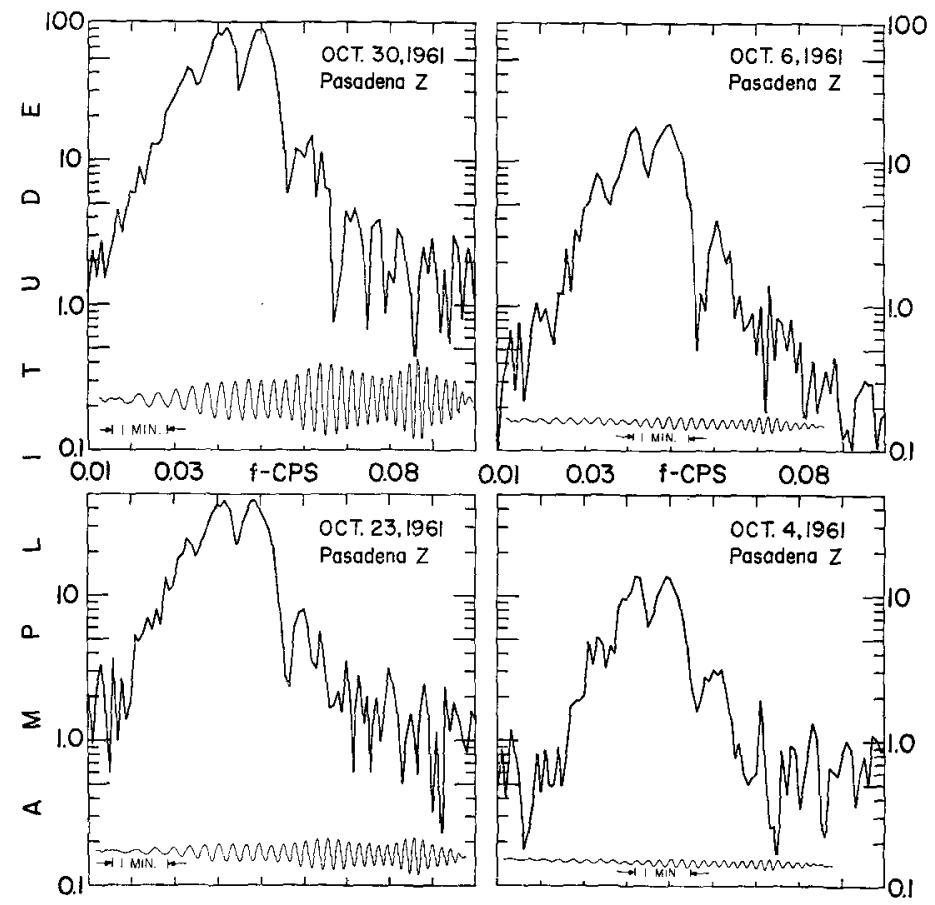

FIG. 20. Amplitude spectra and original records for four nuclear blasts recorded at one station (after Toksöz, Ben-Menahem and Harkrider, 1963).



FIG. 21. Amplitude spectra and original records for one nuclear blast recorded at four stations (after Toksöz, Ben-Menahem and Harkrider, 1963). 
records of various nuclear air blasts at Novaya Zemlya. Here, the source mechanism should be relatively simple. They found that the spectra at any one station were remarkably similar from blast to blast. On the other hand, if the stations were at differing azimuths or at large separations on essentially the same azimuth, the spectra could be quite different. Figures 20 and 21 (from Toksöz, Ben-Menahem, and Harkrider (1963)) show the amplitude spectra of four blasts recorded at one station (Pasadena) and of one blast recorded at four stations. The original seismograms are copied below each spectrum.

\section{Phase Velocities and Structural Interprétation}

No proof has been given that modulation or spectral gaps imply multiple events, but as mentioned above, this is probably the most reasonable explanation. If this interpretation is accepted for the explanation of the observed "beats", then some type of smoothing may exist that will take advantage of the fact that positive and negative phase fluctuations occur relatively equally.

We have noted above that the largest phase changes take place where the amplitudes are lowest; also, the most contamination from noise enters at these points. Hence the phase spectra can be smoothed either by deleting these points or else by weighting points with relatively small amplitudes less strongly in the smoothing process. In addition, between zero frequency and the first amplitude minimum, there is a region where there is an essentially constant time delay. Here, local smoothing is of no help, and only smoothing in the large can eliminate some of this time delay. On the other hand, zero drift, either in the original signal or as a result of imperfect digitization, quite often introduces comparable phase fluctuations at low frequencies. This tends to complicate the problem of smoothing at low frequencies.

We may ask whether phase velocity determinations using modulated records give consistent results when only those portions of the signal occurring before the first minimum in the envelope are used. This is not consistent with the large time errors at low frequencies indicated by the simple theory. The data are sparse concerning this point. There are three possible reasons why contamination may be small in the early portions of the records. First, if the time signals themselves are used in the usual peak-and-trough methods, there may be less contamination of the early phases than if the whole record is Fourier analyzed. Second, all amplitude and phase fluctuations due to a complex source should cancel out if the recording stations are sufficiently close together. Contamination would arise only in the vicinity of the beat because of the relatively low amplitude of the signals at this point. Third, interferences due to path differences may be smaller at longer wavelengths since the earth is relatively more homogeneous at these periods; hence the parameter $a$, as a function of frequency, will be smaller in this range. This is borne out by the fact that most spectra made from records obtained using 15-90 instruments have a relatively smooth character in the 30-100 second range and a ragged character in the 10-30 second range.

Several different types of smoothing have been examined with respect to their ability to reduce phase fluctuations while retaining as much phase information as possible. These are described below. 


\section{Averaging in the small (no weights).}

This is the simplest of all smoothing procedures. The phases at an odd number, $2 N-1$, of contiguous points are averaged to give a new value for the center point. As this filter is moved through all the data, a new set is generated. The number of phase points in the smooth set of data is less than that of the raw data; $N$ points are deleted from each end. This method tends to reduce the curvature of the phase function as well as smooth out local irregularities.

2. Averaging in the small (weighted).

This method is similar to that above but, in addition, the data are weighted proportionally to the spectral amplitudes. At first sight, it would appear that this method would be superior to Method 1, but the scatter in the raw phase data was actually made worse when weights were introduced.

3. Least squares quadratic in the small (weighted).

A quadratic is passed through a contiguous set of phases using a least squares technique and weighting the phases according to the spectral amplitudes. A resultant phase value is computed for the center of the set. Again as this filter is moved through the phase data a new set is generated and again the number of phase points in the new is less than that of the old. Local irregularities are well smoothed, but where the number of points used in the set is increased such that the influence of phase discontinuities is nullified, a prohibitive amount of data is lost at the low frequency end of the spectrum.

4. Least squares polynomial in the large (weighted).

This method follows the data somewhat less well than Methods 1 or 3 above, but all local irregularities are removed. A polynomial of the type $a_{1} \omega+a_{2} \omega^{2}+\cdots$ fitted to the phase differences between two stations seems to follow the data well enough to leave the effects of structure, yet removes the irregularities due to the phase fluctuations. Since the polynomial is fitted to all the data, the number of new phase points is the same as the old. The polynomial contains no constant term inasmuch as it is fitted to phase differences. This helps smooth out irregularities at the low frequency end of the spectrum.

A quantitative basis for selecting weights for these methods has not been obtained. It would seem that the weights should be proportional to some monotonically increasing function of spectral amplitude, i.e., the values of phase should be given less weight when the signal power is low. In the analysis illustrative of the method, the spectral amplitudes themselves were used, although a logarithmic or power function might have been used as well.

As an example of smoothing, we have chosen for a phase velocity determination the event of 9 March 1961. The raw phase data are shown in figure 19. Irregularities of the phase data are often communicated with considerable violence to the resulting phase velocity curve. For example, an 0.05 cycle error in phase at a period of 60 seconds gives rise to a three second error in time determination; at 20 seconds a similar error in phase gives rise to a one second error in time. Depending upon the transit time across the seismic network, the errors in time due to phase errors may or may not be significant. The mean dimension of the Alpine network was of the order of $250 \mathrm{~km}$. At 60 seconds period, an 0.05 cycle error in phase determination will correspond to a $0.2 \mathrm{~km} / \mathrm{sec}$ error in the phase velocity across the net. 
A phase velocity determination in the triangle Besançon-Stuttgart-Oropa for the 9 March 1961 event is shown in figure 22; the reference grid is the same as that used by Press (1956) derived from African group velocities. The dots represent phase velocity determinations using the raw phases of figure 19; near 19 seconds, the period of great uncertainty in phase at Besançon and Stuttgart, the phase velocities fluctuate by about $0.7 \mathrm{~km} / \mathrm{sec}$. The curves identified as 21 Box and 11 Box are the result of smoothing the phases by Method 1 using averages of 21 points and 11 points, respectively. The curve $21 \mathrm{WLS}$ is the result of sliding a least squares quadratic of 21 points along the data, weighted according to the spectral amplitudes (Method 3). The curve P5WLS is the result of fitting least squares polynomials,

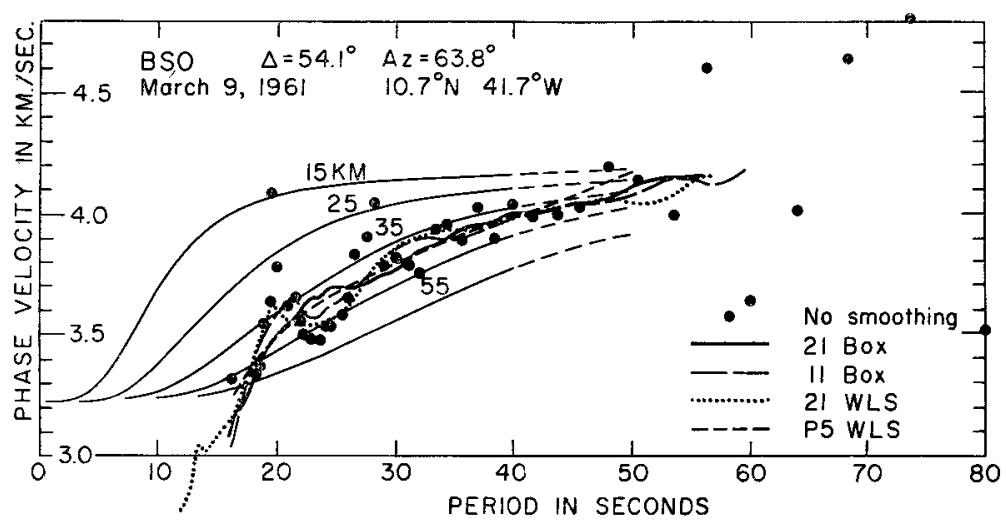

Fig. 22. Results of various methods of smoothing in determination of phase velocity across the tripartite network Besançon-Oropa-Stuttgart for the event of 9 March 1961. The dots represent phase velocities calculated from the phases of Fig. 19.21 Box and 11 Box are the results of smoothing by averages of 21 points and 11 points, respectively. 21 WLS is the result of smoothing by a weighted least squares quadratic of 21 points sliding along the data. P5WLS is the result of smoothing by fitting a weighted least squares polynomial of order five to phase differences.

weighted according to the spectral amplitudes, to the two sets of phase differences Stuttgart minus Besançon and Oropa minus, Besançon (Method 4). It can be seen that it is indeed possible to retain evidence of earth structure in the phase velocity curves and to reduce the effect of interference by the use of smoothing.

It should be remarked that these Alpine data are used here as an illustration; these calculations are not to be taken as a final determination of the structure of the Alps. This structure will be analyzed in a future paper.

\section{Conclusions}

It has been shown that interference of dispersive wave trains can be caused by the nearly simultaneous arrival at a given point of

1) Two or more similar trains separated in time.

2) Two or more almost similar trains separated in time.

3) A continuous superposition of similar events.

4) Two or more trains which have traveled over paths of slightly different lengths but with the same transmission characteristics. 
The word "similar" here is used to mean "identical" except for a constant amplitude factor. In addition, more complex cases could be examined including such complicating factors such as dissimilar transmission characteristics or frequency dependent reflection or refraction coefficients.

Two cases of types 1) or 2) have been observed; twin earthquakes occurring on 9 April 1961 at Hollister, California, and twin earthquakes on 14 June 1962 in the Aleutian Islands. In the second case, rough estimates of the relative magnitudes of the time events and their time separations were obtained from the amplitude and phase spectra of the superposed Rayleigh wave trains. These were comparable to relative magnitudes obtained by measurements in the time domain on both body and surface waves. It was also demonstrated that the phase and amplitude fluctuations due to the multiple source mechanism were essentially the same at all stations of the observing network and that differences in phase cancelled, to first order.

In the example of 9 March 1961, the interference was attributed to multipath propagation since the amplitude minima and phase jumps in the Fourier spectra were not common to all stations. Other authors have noted the same phenomenon in connection with nuclear air blasts, where there was remarkable similarity in the records and spectra when the same epicenter and receiving station are concerned, but there were large differences when recording the same event at widely separated stations.

An analysis was made of the phase velocities across a seismic network near and in the Alps, a structurally complicated region, of the earthquake of March 9, 1961 and the effects of several smoothing techniques are discussed. It appears to be possible to reduce considerably the effects due to interferences while preserving the effects of structure. Further work is necessary to evaluate the efficiency of these smoothing techniques.

Although the evaluation of the relative amplitudes and time separation of multiple events is not very precise, there exists a possibility that the influence of large geologic features such as mountain ranges and continental margins as seismic scatterers can be determined by use of the techniques described here. On the other hand, separation in the time domain of interfering events is very difficult unless the events are quite widely separated; the variable filter technique described here is not recommended for this purpose.

\section{ACKNOWLEDGMENTS}

This research was supported by grant AF-AFOSR-26-63 of the Air Force Office of Scientific Research as part of the Advanced Research Projects Agency project Vela.

We would like to thank Drs. M. Nafi Toksoz, Ari Ben-Menahem and David Harkrider for their kind permission to use figures 20 and 21 . We would like to acknowledge the assistance of Mr. Laszlo Lenches in the preparation of graphical material.

\section{References}

Ben-Menahem, A.

1961. "Radiation of Seismic Surface-Waves from Finite Moving Sources," Bull. Seism. Soc. Am., 51: 401-435.

Ben-Menahem, A., and M. N. Toksöz

1962. "Source Mechanism from Spectra of Long-Period Seismic Surface Waves, 1. The Mongolian Earthquake of December 4, 1957," Journ. of Geophys. Res., 67: 1943-1955. 
1963a. "Source Mechanism from Spectra of Long-Period Seismic Surface Waves, 2. The Kamchatka Earthquake of November 4, 1952," Journ. of Geophys. Res., 68: 52075222 .

1963b. "Source Mechanism from Spectra of Long-Period Seismic Surface Waves, 3. The Alaska Earthquake of July 10, 1958," Bull. Seism. Soc. Am., 53: 905-919.

Pomeroy, P. W.

1963. "Long Period Seismic Waves from Large, Near Surface Nuclear Explosions," Bull. Seism. Soc. Am., 53: 109-149.

Press, F.

1956. "Determination of Crustal Structure from Phase Velocity of Rayleigh WavesPart 1: Southern California," Bull. Geol. Soc. Am., 67: 1647-1658.

Toksöz, M. N., A. Ben-Menahem, and D. G. Harkrider

1963. "Radiation of Seismic Surface Waves from Underground and Atmospheric Nuclear Explosions," (in preparation).

Seismological Laboratory,

California Institute of Technology,

Pasadena, California

(Division of the Geological Sciences, contribution No. 1211)

W. L. Pilant is now at the Dept. of Earth and Planetary Science, University of Pittsburgh, Pittsburgh, Pennsylvania.

Manuscript received October 18, 1963. 